\title{
Recursive Filter with Partial Knowledge on Inputs and Outputs
}

\author{
Jinya $\mathrm{Su}^{1} \quad$ Baibing $\mathrm{Li}^{2} \quad$ Wen-Hua Chen ${ }^{1}$ \\ ${ }^{1}$ Department of Aeronautical and Automotive Engineering, Loughborough University, Loughborough, LE11 3TU, UK \\ ${ }^{2}$ School of Business and Economics, Loughborough University, Loughborough, LE11 3TU, UK
}

\begin{abstract}
This paper investigates the problem of state estimation for discrete-time stochastic linear systems, where additional knowledge on the unknown inputs is available at an aggregate level and the knowledge on the missing measurements can be described by a known stochastic distribution. Firstly, the available knowledge on the unknown inputs and the state equation is used to form the prior distribution of the state vector at each time step. Secondly, to obtain an analytically tractable likelihood function, the effect of missing measurements is broken down into a systematic part and a random part, and the latter is modeled as part of the observation noise. Then, a recursive filter is obtained based on Bayesian inference. Finally, a numerical example is provided to evaluate the performance of the proposed methods.
\end{abstract}

Keywords: Bayesian inference, Kalman filter, missing measurements, state estimation, unknown inputs.

\section{Introduction}

State estimation for discrete-time stochastic linear systems has received much attention in the past few decades due to its versatile applications in a wide range of areas, such as network control system, transportation management, environmental applications, etc. ${ }^{[1-4]}$ Recently, the state filtering problem with sensor missing measurements (also termed as intermittent sensor fault, packet loss or drop-out, etc.) is also drawing considerable research interests, especially in the field of fault detection, network control system, etc. ${ }^{[5-11]}$ In these fields, in addition to environment noise and sensor noise, the sensor measurements usually subject to information loss for multiple reasons, such as sensor failures, network congestion, accidental loss of some collected data, etc. ${ }^{[5-7]}$ To describe this phenomenon, some scholars multiply the measurement output matrix by a gain matrix whose entries are stochastic variables (see [5] for Bernoulli distributed variables or [6] for any distributed variables on the interval $[0,1]$ with known expectation and variance).

However, in the aforementioned literature, the unforeseen inputs into the channel of state transition dynamics are usually modeled as process noise with known description (e.g., mean and covariance are known). Although it can account for the effects of un-modeled dynamics (such as system mismatch, external disturbances, parameter uncertainties, input noises, etc.) to some extent, it has been pointed out in

Regular paper

Special Issue on Recent Advance in Automation and Computing Manuscript received December 18, 2013; accepted September 24 2014

This work was jointly funded by UK Engineering and Physical Sciences Research Council (EPSRC) and BAE System (EP/H501401/1).

Recommended by Associate Editor Yi Cao

(C) Institute of Automation, Chinese Academy of Science and Springer-Verlag Berlin Heidelberg 2015
$[4,5]$ that this method is not an ideal solution in general, especially when little is known about the input variables, it may result in a poor filtering performance in face of abrupt unknown inputs ${ }^{[4]}$. To this end, some scholars describe the unforeseen inputs to the system using completely unknown inputs $^{[4,12]}$ (also termed as external disturbances ${ }^{[13-16]}$ ). It is well known that in the field of control design, both control performance and disturbance rejection property can be substantially improved if the knowledge on unknown inputs or disturbances can be obtained (or approximately obtained) and incorporated into the control scheme design (termed as disturbance observer based control $(\mathrm{DOBC}))^{[13-16]}$. Similar philosophy can be also applied in the field of filter design, especially when the knowledge on unknown inputs is directly available. For example, in the field of population estimation, traffic density estimation, etc. ${ }^{[12]}$, some knowledge on unknown inputs is directly available at an aggregate level. For the filtering problem that knowledge on inputs in the channel of state dynamics is available only at an aggregate (or set) level, [12] has proposed a unified filtering approach to incorporate this type of knowledge. It is also shown that this approach can include two extreme scenarios as its special cases, i.e., the filter where all inputs are unknown (i.e., the scenario investigated in $[4,17,18]$, etc.) and the filter where the inputs are completely available (i.e., the classical Kalman filter $\left.{ }^{[19]}\right)$. However, the developed filter relies on the assumption that the measurement signals are perfectly obtained, subject to sensor noise.

In this paper, we consider the problem of state estimation for discrete-time stochastic linear systems, where additional knowledge on unknown inputs is available at an aggregate level, and the sensors are subject to multiple missing measurements described by a known stochastic distribution. Firstly, similar to [12], we incorporate a Bayesian approach to pool the prior knowledge about the system dynamics and 
unknown inputs at the aggregate level into state prior prediction. Secondly, we approximate the measurement likelihood function by pooling the effect of missing measurements into the measurement noise. Finally, a recursive optimal filter is obtained based on Bayesian inference, which is suitable for online applications. It should be noted that a part of the results has been reported in [20].

The rest of the paper is organized as follows. The problem considered in this paper is formulated in Section 2. The main results are provided in Section 3. Numerical example and simulation comparison are given in Section 4 to evaluate the performance of the proposed method. Section 5 is devoted to the conclusions of the paper and possible future research directions.

Notations. Throughout this paper, $\mathbf{R}^{m \times n}$ denotes the linear space of all $m \times n$ matrices over the real field. For any $K \in \mathbf{R}^{m \times n}$, the symbols $K^{\mathrm{T}}$ denotes the transpose of $K$, while $K^{+}$stands for the Penrose-Moore inverse of $K$. $\circ$ denotes the Hadamard product defined as $[A \circ B]_{i j}=A_{i j} \times B_{i j} \cdot \operatorname{diag}\left\{x_{1}, x_{2}, \cdots, x_{n}\right\}$ stands for a block-diagonal matrix with elements $x_{1}, x_{2}, \cdots, x_{n}$ on the diagonal.

\section{Problem formulation}

Consider the linear discrete-time stochastic time-varying system with partial knowledge on both unknown inputs and outputs in the following form:

$$
\begin{aligned}
& x_{k+1}=A_{k} x_{k}+G_{k} d_{k}+w_{k} \\
& y_{k}=\Xi_{k} C_{k} x_{k}+v_{k}
\end{aligned}
$$

where $x_{k} \in \mathbf{R}^{n}$ is the state vector, $d_{k} \in \mathbf{R}^{p}$ is the input vector, and $y_{k} \in \mathbf{R}^{p}$ is the measurement vector at each time step. The process noise $w_{k} \in \mathbf{R}^{n}$ and the measurement noise $v_{k} \in \mathbf{R}^{p}$ are assumed to be mutually independent, and each follows a Gaussian distribution with zero mean and a known covariance matrix, $Q_{k}=\mathrm{E}\left[\omega_{k} \omega_{k}^{\mathrm{T}}\right]>0$ and $V_{k}=\mathrm{E}\left[v_{k} v_{k}^{\mathrm{T}}\right]>0$, respectively. $A_{k}, G_{k}$ and $C_{k}$ are known matrices and it is assumed that $G_{k}$ has a full-column rank. Following the notation of [6], $\Xi_{k}=\operatorname{diag}\left\{\xi_{k}^{1}, \xi_{k}^{2}, \cdots, \xi_{k}^{p}\right\}$ is used to account for the missing measurements where the mutually uncorrelated (in $k$ and $i$ ) random variables $\xi_{k}^{i} \in \mathbf{R}(i=1,2, \cdots, p)$ have a probability density function $p_{k}^{i}$ on the interval $[0,1]$ with known mathematical expectation $\mu_{k}^{i}$ and variance $\left(\sigma_{k}^{i}\right)^{2}(i=1,2, \cdots, p)$.

In addition, $\xi_{k}^{i}$ is assumed to be independent of $w_{k}, v_{k}$ and $x_{0}$, respectively. The initial state $x_{0}$ is independent of $w_{k}$ and $v_{k}$ with a known mean $\hat{x}_{0}$ and covariance matrix $P_{0}>0$. Following [12], we investigate the scenario where the input vector $d_{k}$ is not fully observed at the level of interest, but available at an aggregate level. Specifically, let $D_{k}$ be a $q_{k} \times m$ known matrix with $0 \leq q_{k} \leq m$ and $F_{0 k}$ be an orthogonal complement of $D_{k}^{\mathrm{T}}$. It is assumed that the input data is available only on some linear combinations $D_{k} d_{k}$ as

$$
r_{k}=D_{k} d_{k}
$$

where $r_{k}$ is observed at each time step $k$ and no information about $\delta_{k}=F_{0 k}^{\mathrm{T}} d_{k}$ is available. Hence, $\delta_{k}$ is assumed to have a noninformative distribution, i.e., all values of $\delta_{k}$ are equally likely to occur:

$$
f\left(\delta_{k}\right) \propto 1
$$

Here, the matrix $D_{k}$ characterizes the availability (or knowledge) of input information at each time step $k$. It includes two extreme scenarios that are usually considered: 1) When $q_{k}=0$, i.e., no knowledge on the input variables is available, it reduces to the scenario investigated in $[4,18]$. 2) When $q_{k}=m$ and $D_{k}$ is an identity matrix, it is the scenario where the complete input information is available, that the classical Kalman filter can be applied.

The objective of this paper is to design a recursive filter which can estimate the system state $x_{k}$ based on the initial estimate $\hat{x}_{0}$, the sequence of measurements $Y_{k}=$ $\left[y_{0}, y_{1}, \cdots, y_{k}\right]$ when the unknown inputs are only available at an aggregate level and the system outputs are subject to multiple missing sensor measurements.

\section{Main results}

In this section, the recursive filter is firstly derived in Subsection 3.1 and the relationship of the proposed filter with the existing results is considered in Subsection 3.2.

\subsection{Filter design}

Following the method in [12], we will incorporate a Bayesian approach: At each step $k$, the state equations, combined with the previous state estimates, provide prior knowledge about the current state vector, whereas the observation equations specify the likelihood function. The updated estimate of the state vector is obtained from the posterior distribution. The key idea of this paper is to work out the likelihood function by breaking down the effect of missing measurement and modelling the associated uncertainties as part of the measurement noise.

First, we consider the prior prediction distribution using the state equations (1). Suppose that at time $k-1$, we have obtained the posterior distribution of $x_{k-1}$ for given $Y_{k-1}$, i.e., $x_{k-1} \sim N\left(\hat{x}_{k-1 \mid k-1}, P_{k-1 \mid k-1}\right)$. Note that this is true for $k=1$ with $\hat{x}_{0 \mid 0}=\hat{x}_{0}$ and $P_{0 \mid 0}=P_{0}$. Then from (1), we can obtain the prior distribution at time step $k$ :

$$
\begin{aligned}
& x_{k} \sim N\left(\hat{x}_{k \mid k-1}, P_{k \mid k-1}\right) \\
& \hat{x}_{k \mid k-1}=A_{k-1} \hat{x}_{k-1 \mid k-1}+G_{k-1} d_{k-1} \\
& P_{k \mid k-1}=A_{k-1} P_{k-1 \mid k-1} A_{k-1}^{\mathrm{T}}+Q_{k-1} .
\end{aligned}
$$

It should be noticed that the above prior distribution is unavailable due to the presence of unknown inputs $d_{k}$. To this end, we follow [12] and transform the state vector into two parts: One part has the prior distribution involving the unknown inputs $\delta_{k}$, whereas the other part does not depend on the unknown inputs, which will be used as the prior prediction for further inference. Specifically, let 
$\tilde{D}_{k}=\left[\begin{array}{cc}D_{k} & O \\ O & I\end{array}\right]$ be a $\left(q_{k}+n-m\right) \times n$ matrix and define
$M_{k}$ as

$$
M_{k}= \begin{cases}\tilde{D}_{k} \Omega_{k}^{-1}, & \text { if } G_{k} \neq 0 \\ I, & \text { if } G_{k}=0\end{cases}
$$

where $\Omega_{k}=\left[G_{k}, G_{k}^{\perp}\right]$, and $G_{k}^{\perp}$ denotes an orthogonal compliment of $G_{k}$. As shown in [12], the transformed state vector $s_{k}=M_{k-1} x_{k}$ leads to the prior distribution required for Bayesian inference:

$$
s_{k} \sim N\left(M_{k-1} A_{k-1} \hat{x}_{k-1 \mid k-1}+\tilde{r}_{k-1}, \tilde{P}_{k \mid k-1}\right) .
$$

Note that in (4), the covariance matrix $\tilde{P}_{k \mid k-1}=$ $M_{k} P_{k \mid k-1} M_{k}^{\mathrm{T}}$ is available and $\tilde{r}_{k}=\left[r_{k}^{\mathrm{T}}, O^{\mathrm{T}}\right]^{\mathrm{T}}$ can be obtained directly from (4). So the prediction has been obtained.

Next, we consider the likelihood function. Due to the presence of missing measurements, the likelihood function becomes very complicated. In order to derive a recursive filter, we re-arrange the observation equation in the following form:

$$
y_{k}=\bar{\Xi}_{k} C_{k} x_{k}+\left(\Xi_{k}-\bar{\Xi}_{k}\right) C_{k} x_{k}+v_{k}
$$

where $\bar{\Xi}_{k}:=\mathrm{E}\left\{\Xi_{k}\right\}:=\operatorname{diag}\left\{\mu_{k}^{1}, \mu_{k}^{2}, \cdots, \mu_{k}^{p}\right\}$. Note that $\mathrm{E}\left\{\Xi_{k}-\bar{\Xi}_{k}\right\}=\mathrm{E}\left\{\Xi_{k}\right\}-\mathrm{E}\left\{\bar{\Xi}_{k}\right\}=0$, which results in $\mathrm{E}\left\{\left(\Xi_{k}-\bar{\Xi}_{k}\right) C_{k} x_{k} \mid x_{k}\right\}=0$. It should be noticed that $\left(\Xi_{k}-\bar{\Xi}_{k}\right) C_{k} x_{k}$ generally does not follow a Gaussian distribution, but to make the problem tractable, we treat the zero-mean random variable $\left(\Xi_{k}-\bar{\Xi}_{k}\right) C_{k} x_{k}$ as part of the measurement error and assume that it approximately follows a Gaussian distribution with zero mean and covariance of $\operatorname{var}\left\{\left(\Xi_{k}-\bar{\Xi}_{k}\right) C_{k} x_{k}\right\}$.

According to the property of conditional expectation, the covariance of $\left(\Xi_{k}-\bar{\Xi}_{k}\right) C_{k} x_{k}$ is governed by

$$
\begin{aligned}
P_{x}= & \mathrm{E}\left\{\left(\Xi_{k}-\Xi_{k}\right) C_{k} x_{k} x_{k}^{\mathrm{T}} C_{k}^{\mathrm{T}}\left(\Xi_{k}-\bar{\Xi}_{k}\right) \mid x_{k}\right\}= \\
& \breve{\Xi}_{k} \circ\left(C_{k} x_{k} x_{k}^{\mathrm{T}} C_{k}^{\mathrm{T}}\right)
\end{aligned}
$$

where

$$
\breve{\Xi}_{k}:=\operatorname{diag}\left\{\left(\sigma_{k}^{1}\right)^{2},\left(\sigma_{k}^{2}\right)^{2}, \cdots,\left(\sigma_{k}^{p}\right)^{2}\right\} .
$$

Hadamard product $\circ$ is defined in Section 1. Similar to [6], the second equation of (5) is obtained using the theorem in [21].

It should be highlighted that, due to the presence of partially observed inputs $d_{k-1}$, the dynamics of $x_{k}$ and hence $x_{k} x_{k}^{\mathrm{T}}$ are no longer available. Consequently, the methods in $[5,8]$ are not directly applicable. Instead, we suggest two simple solutions to tackling the problem. The first one is to estimate $x_{k} x_{k}^{\mathrm{T}}$ based on the available $\mathrm{E}\left\{x_{k-1} x_{k-1}^{\mathrm{T}}\right\}$ which equals to the sum of the covariance of $x_{k-1}$ and $\hat{x}_{k-1} \hat{x}_{k-1}^{\mathrm{T}}$. The other solution is to estimate $x_{k} x_{k}^{\mathrm{T}}$ based on the prior estimation of $x_{k}{ }^{[6]}$ which in our case can be obtained using the prior estimation of $M_{k-1} x_{k}$ due to the presence of unknown inputs $d_{k}$. We outline the second approach as follows:
Firstly, we can obtain from (4) that

$$
\begin{aligned}
P_{M x}= & \mathrm{E}\left\{M_{k-1} x_{k} x_{k}^{\mathrm{T}} M_{k-1}^{\mathrm{T}} \mid x_{k-1}\right\}= \\
& \mathrm{E}\left\{M_{k-1} e_{k} e_{k}^{\mathrm{T}} M_{k-1}^{\mathrm{T}} \mid x_{k-1}\right\}+ \\
& \mathrm{E}\left\{M_{k-1} \hat{x}_{k} \hat{x}_{k}^{\mathrm{T}} M_{k-1}^{\mathrm{T}} \mid x_{k-1}\right\}
\end{aligned}
$$

where $M_{k-1} \hat{x}_{k}$ and $M_{k-1} e_{k}$ are governed by

$$
\begin{aligned}
& M_{k-1} \hat{x}_{k}=M_{k-1} A_{k-1} \hat{x}_{k-1 \mid k-1}+\tilde{r}_{k-1} \\
& M_{k-1} e_{k}=M_{k-1} x_{k}-M_{k-1} \hat{x}_{k} .
\end{aligned}
$$

Noting that the covariance of $M_{k-1} x_{k}$ is given by $\tilde{P}_{k \mid k-1}$ and in conjunction with (7), we obtain that (6) reduces to

$$
\begin{aligned}
P_{M x}= & \tilde{P}_{k \mid k-1}+\left(M_{k-1} A_{k-1} \hat{x}_{k-1 \mid k-1}+\tilde{r}_{k-1}\right) \times \\
& \left(M_{k-1} A_{k-1} \hat{x}_{k-1 \mid k-1}+\tilde{r}_{k-1}\right)^{\mathrm{T}} .
\end{aligned}
$$

From (6), we can obtain $M_{k-1} \mathrm{E}\left\{x_{k} x_{k}^{\mathrm{T}}\right\} M_{k-1}^{\mathrm{T}}=P_{M x}$. We solve this equation for $\mathrm{E}\left\{x_{k} x_{k}^{\mathrm{T}}\right\}$ by choosing the MoorePenrose generalized inverse of $M_{k-1}$ as

$$
\mathrm{E}\left\{x_{k} x_{k}^{\mathrm{T}}\right\}=M_{k-1}^{+} P_{M x}\left(M_{k-1}^{+}\right)^{\mathrm{T}} .
$$

If we estimate $x_{k} x_{k}^{\mathrm{T}}$ using $\mathrm{E}\left\{x_{k} x_{k}^{\mathrm{T}}\right\}$, then (5) will be governed by

$$
\left.P_{x}=\breve{\Xi}_{k} \circ\left[C_{k} M_{k-1}^{+} P_{M x}\left(M_{k-1}^{+}\right)^{\mathrm{T}} C_{k}^{\mathrm{T}}\right)\right] .
$$

This means the covariance of $\left(\Xi_{k}-\bar{\Xi}_{k}\right) C_{k} x_{k}+v_{k}$ can be approximated as $\bar{V}_{k}=P_{x}+V_{k}$.

Now, if we approximate the effect of $\left(\Xi_{k}-\bar{\Xi}_{k}\right) C_{k} x_{k}$ on the likelihood function using a Gaussian distribution, output dynamics can be expressed as

$$
y_{k}=\bar{\Xi}_{k} C_{k} x_{k}+\bar{v}_{k}
$$

where $\bar{v}_{k}$ satisfies a Gaussian distribution governed by $\bar{v}_{k} \sim$ $N\left(0, \bar{V}_{k}\right)$.

Until now, the problem of state estimation for linear stochastic systems subject to both partially observed inputs and multiple missing measurements (1) has been transformed into the one without missing measurements. The likelihood function can thus be written as

$$
L\left(x_{k} \mid y_{k}\right) \propto \exp \left(-\left[y_{k}-\bar{\Xi}_{k} C_{k} x_{k}\right]^{\mathrm{T}}\left(\bar{V}_{k}\right)^{-1}\left[y_{k}-\bar{\Xi}_{k} C_{k} x_{k}\right]\right) .
$$

Now both the prior distributions (3), (4) and likelihood function (8) do not depend on the unknown inputs, hence we can apply Bayes' theorem to derive the posterior distribution of $x_{k}$. Although Bayesian approach is used here, the obtained filter also falls into the category of linear filters since a linear system with Gaussian noise is considered. The diagram of the system and filter is shown in Fig. 1. The result of the filter is summarized by Theorem 1 . 


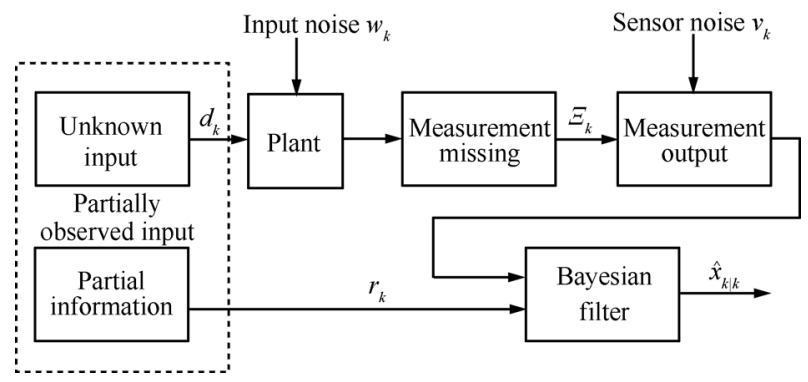

Fig. 1 The diagram of filter structure

Theorem 1. For state space model (1), suppose that the input data is available only at an aggregate level specified by (2), and the matrix

$$
\Pi_{k}=\left[\begin{array}{c}
D_{k-1} \\
\bar{\Xi}_{k} C_{k} G_{k-1}
\end{array}\right]
$$

has a full column-rank. Then, the prior and posterior distributions for $x_{k}$ at any time step $k$ can be obtained sequentially as

1) Posterior of $x_{k-1}$ for given $\left[y_{0}, y_{1}, \cdots, y_{k-1}\right]$ :

$$
x_{k-1} \sim N\left(\hat{x}_{k-1 \mid k-1}, P_{k-1 \mid k-1}\right) .
$$

2) Prior for $s_{k}=M_{k-1} x_{k}$ :

$$
\begin{gathered}
s_{k} \sim N\left(M_{k-1} A_{k-1} \hat{x}_{k-1 \mid k-1}+\tilde{r}_{k-1}, \tilde{P}_{k \mid k-1}\right) \\
\tilde{P}_{k \mid k-1}=M_{k} P_{k \mid k-1} M_{k}^{\mathrm{T}} \\
P_{k \mid k-1}=A_{k-1} P_{k-1 \mid k-1} A_{k-1}^{\mathrm{T}}+Q_{k-1} .
\end{gathered}
$$

3) Posterior of $x_{k}$ for given $Y_{k}=\left[y_{0}, y_{1}, \cdots, y_{k-1}\right]$ :

$$
x_{k} \sim N\left(\hat{x}_{k \mid k}, P_{k \mid k}\right)
$$

where the posterior mean is given by

$$
\begin{gathered}
\hat{x}_{k \mid k}=A_{k-1} \hat{x}_{k-1 \mid k-1}+P_{k \mid k} M_{k-1}^{\mathrm{T}}\left(M_{k-1} P_{k \mid k-1} M_{k-1}^{\mathrm{T}}\right)^{-1} \times \\
\tilde{r}_{k-1}+K_{k}\left(y_{k}-\bar{\Xi}_{k} C_{k} A_{k-1} \hat{x}_{k-1 \mid k-1}\right)
\end{gathered}
$$

and the gain matrix and the posterior variance are given by

$$
\begin{aligned}
K_{k}= & P_{k \mid k} C_{k}^{\mathrm{T}} \bar{\Xi}_{k}^{\mathrm{T}} \bar{V}_{k}^{-1} \\
P_{k \mid k}= & \left\{M_{k-1}^{\mathrm{T}}\left(M_{k-1} P_{k \mid k-1} M_{k-1}^{\mathrm{T}}\right)^{-1} M_{k-1}+\right. \\
& \left.C_{k}^{\mathrm{T}} \bar{\Xi}_{k}^{\mathrm{T}} \bar{V}_{k}^{-1} \bar{\Xi}_{k} C_{k}\right\}^{-1} .
\end{aligned}
$$

Considering the above equation involves the inverse of an $n \times n$ matrix which may require high computation for large $n$, we provide an alternative for the computation of the filter. Let $F_{k}$ denote an orthogonal complement of $M_{k}^{\mathrm{T}}$, where $F_{k}$ can be constructed as $F_{k}=\Omega_{k}\left[\begin{array}{c}F_{0 k} \\ O\end{array}\right]$. From Lemma 2 of [12], we can express $K_{k}$ and $P_{k \mid k}$ in a recursive form as

$$
\begin{aligned}
& K_{k}=P_{k \mid k-1} C_{k}^{\mathrm{T}} \bar{\Xi}_{k}^{\mathrm{T}} H_{k}^{-1}+ \\
& \quad\left[F_{k-1}-P_{k \mid k-1} C_{k}^{\mathrm{T}} \bar{\Xi}_{k}^{\mathrm{T}} H_{k}^{-1} \bar{\Xi}_{k} C_{k} F_{k-1}\right] \times \\
& \quad\left[F_{k-1}^{\mathrm{T}} C_{k}^{\mathrm{T}} \bar{\Xi}_{k}^{\mathrm{T}} H_{k}^{-1} \bar{\Xi}_{k} C_{k} F_{k-1}\right]^{-1} F_{k-1}^{\mathrm{T}} C_{k}^{\mathrm{T}} \bar{\Xi}_{k}^{\mathrm{T}} H_{k}^{-1} \\
& P_{k \mid k}=P_{k \mid k-1}-P_{k \mid k-1} C_{k}^{\mathrm{T}} \bar{\Xi}_{k}^{\mathrm{T}} H_{k}^{-1} \bar{\Xi}_{k} C_{k} P_{k \mid k-1}+\left[F_{k-1}-\right. \\
& \left.P_{k \mid k-1} C_{k}^{\mathrm{T}} \bar{\Xi}_{k}^{\mathrm{T}} H_{k}^{-1} \bar{\Xi}_{k} C_{k} F_{k-1}\right]\left[F_{k-1}^{\mathrm{T}} C_{k}^{\mathrm{T}} \bar{\Xi}_{k}^{\mathrm{T}} H_{k}^{-1} \bar{\Xi}_{k} C_{k} F_{k-1}\right]^{-1} \times \\
& {\left[F_{k-1}-P_{k \mid k-1} C_{k}^{\mathrm{T}} \bar{\Xi}_{k}^{\mathrm{T}} H_{k}^{-1} \bar{\Xi}_{k} C_{k} F_{k-1}\right]^{\mathrm{T}}}
\end{aligned}
$$

with $H_{k}=\bar{\Xi}_{k} C_{k} P_{k \mid k-1} C_{k}^{\mathrm{T}} \bar{\Xi}_{k}^{\mathrm{T}}+\bar{V}_{k}>0$.

\subsection{Relationship with the existing results}

The developed filter is closely related to some existing filters, which is briefly discussed below.

Case 1. If no missing measurements occur in the output dynamics, i.e., the expectation of random variables $\xi_{k}^{i} \in \mathbf{R}(i=1,2, \cdots, p)$ are $\mu_{k}^{i}=1$ and the variance of them are $\left(\sigma_{k}^{i}\right)^{2}$, then $\bar{V}_{k}$ will reduce to $V_{k}$. Obviously, the gain matrix $K_{k}$, the posterior variance $P_{k \mid k}$ and posterior mean (9) will reduce to the ones in [12].

Case 2. In the absence of partially observed inputs $d_{k}$, i.e., $G_{k}=0$, it can be verified that (9), (10) and (11) will reduce to

$$
\begin{aligned}
& \hat{x}_{k \mid k}=A_{k-1} \hat{x}_{k-1 \mid k-1}+K_{k}\left(y_{k} \bar{\Xi}_{k} C_{k} A_{k-1} \hat{x}_{k-1 \mid k-1}\right) \\
& K_{k}=P_{k \mid k} C_{k}^{\mathrm{T}} \bar{\Xi}_{k}^{\mathrm{T}} H_{k}^{-1} \\
& P_{k \mid k}=P_{k \mid k-1}-P_{k \mid k-1} C_{k}^{\mathrm{T}} \bar{\Xi}_{k}^{\mathrm{T}} H_{k}^{-1} \bar{\Xi}_{k} C_{k} P_{k \mid k-1}
\end{aligned}
$$

with $H_{k}=\bar{\Xi}_{k} C_{k} P_{k \mid k-1} C_{k}^{\mathrm{T}} \bar{\Xi}_{k}^{\mathrm{T}}+\bar{V}_{k}>0$.

These recursive formulas are identical to the filter in [6] for the linear case.

\section{Simulation example}

In this section, we use a simple numerical example to illustrate the developed filters. Special attention has been paid to showing the filtering performance improvement by incorporating the knowledge on unknown inputs.

\subsection{Simulation scenario}

Consider system (1) with

$$
A_{k}=\left[\begin{array}{ccccc}
0.5 & 2 & 0 & 0 & 0 \\
0 & 0.2 & 1 & 0 & 1 \\
0 & 0 & 0.3 & 0 & 1 \\
0 & 0 & 0 & 0.7 & 1 \\
0 & 0 & 0 & 0 & 0.1
\end{array}\right]
$$




$$
\begin{gathered}
G_{k}=\left[\begin{array}{ccc}
1 & 0 & 0 \\
0 & 0 & 0 \\
0 & 1 & 0 \\
0 & 0 & 0 \\
0 & 0 & 0.1
\end{array}\right] \\
Q_{k}=10^{-3} \times\left[\begin{array}{ccccc}
1 & 0 & 0 & 0 & 0 \\
0 & 1 & 0.5 & 0 & 0 \\
0 & 0.5 & 1 & 0 & 0 \\
0 & 0 & 0 & 1 & 0 \\
0 & 0 & 0 & 0 & 1
\end{array}\right] \\
R_{k}=10^{-2} \times\left[\begin{array}{ccccc}
1 & 0 & 0 & 0.5 & 0 \\
0 & 1 & 0 & 0 & 0.3 \\
0 & 0.5 & 1 & 0 & 0 \\
0.5 & 0 & 0 & 1 & 0 \\
0 & 0.3 & 0 & 0 & 1
\end{array}\right] .
\end{gathered}
$$

and $C_{k}=I_{5}$ is a $5 \times 5$ identity matrix. Similar systems were investigated in many previous studies ${ }^{[17,20]}$.

The data used in this example was simulated using the system (1). To investigate the problem of partially observed inputs, we further assume that the input variables are observed at an aggregate level (2) with

$$
D_{k}=\left[\begin{array}{lll}
1 & 0 & 1 \\
0 & 1 & 1
\end{array}\right] .
$$

For the input vector $d_{k}=\left[d_{1 k}, d_{2 k}, d_{3 k}\right]^{\mathrm{T}}$, it means that the information on $d_{1 k}+d_{3 k}$ and $d_{2 k}+d_{3 k}$ is available at each time step although each individual input is unknown. The dynamics of $d_{1 k}, d_{2 k}$ and $d_{3 k}$ in simulation are shown in Fig. 2.
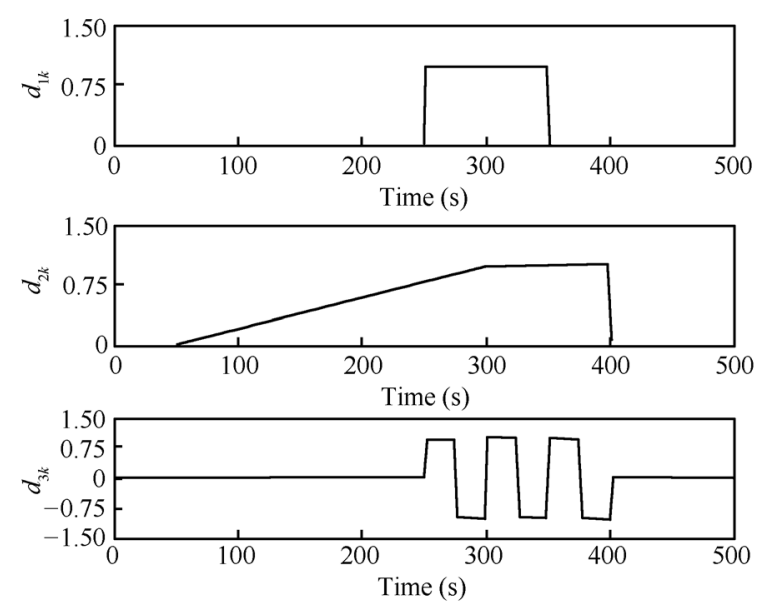

Fig. 2 Dynamic of unknown inputs $d_{1 k}, d_{2 k}$ and $d_{3 k}$

To simulate the occurrence of the multiple missing measurements, we suppose $\xi_{k}^{i}$ of $\Xi_{k}=\operatorname{diag}\left\{\xi_{k}^{1}, \cdots, \xi_{k}^{5}\right\}$ follows a Bernoulli distribution as below:

$$
p\left(\xi_{k}^{i}\right)=\left\{\begin{array}{l}
0.05, \text { if } \xi_{k}^{i}=0 \\
0.95, \text { if } \xi_{k}^{i}=1
\end{array}\right.
$$

for $i=1, \cdots, 5$, which means the expectation of $\xi_{k}^{i}$ is 0.95 and the variance is 0.0475 . The phenomenon of missing measurements is shown in Fig. 3.

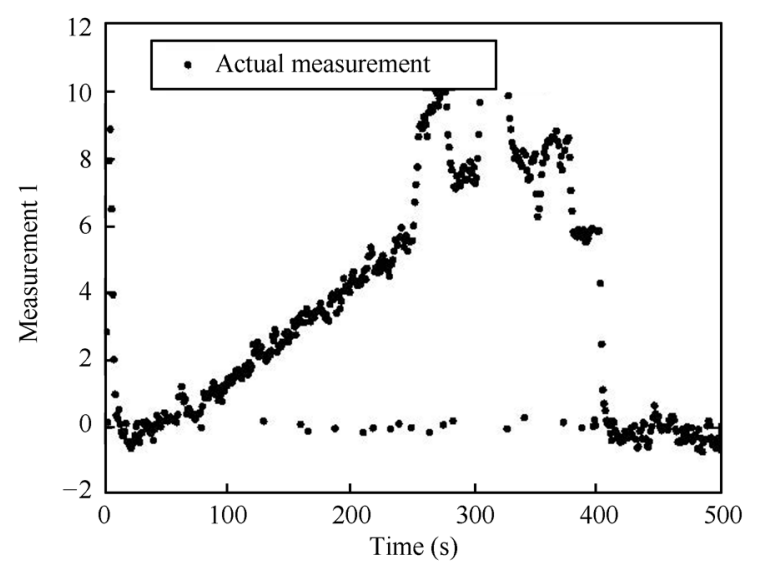

Fig. 3 Phenomenon of missing measurements

We applied the proposed recursive formulas in this paper to estimate the state vector at each time step, where the approach of estimating $x_{k} x_{k}^{\mathrm{T}}$ by $\mathrm{E}\left\{x_{k-1} x_{k-1}^{\mathrm{T}}\right\}$ is termed method 1 , while the other approach is termed method 2 .

\subsection{Simulation comparisons}

For the purpose of comparison, we firstly show the results of the classical Kalman filter, which only takes into account the knowledge of sensor missing measurement and overlooks the effect of unknown inputs. Similar technique to the proposed method 1 based filter has been used to tackle the problem of multiple sensor missing measurements. The first state estimation (upper graphic) and estimation error (lower graphic) are shown in Fig. 4.

It can be seen from Fig. 4 that, in the presence of unknown inputs (from $50 \mathrm{~s}$ to $400 \mathrm{~s}$ ), the classical Kalman filter will obtain a biased estimation. This is because the unknown input dynamics have not been taken into account when designing the filter.
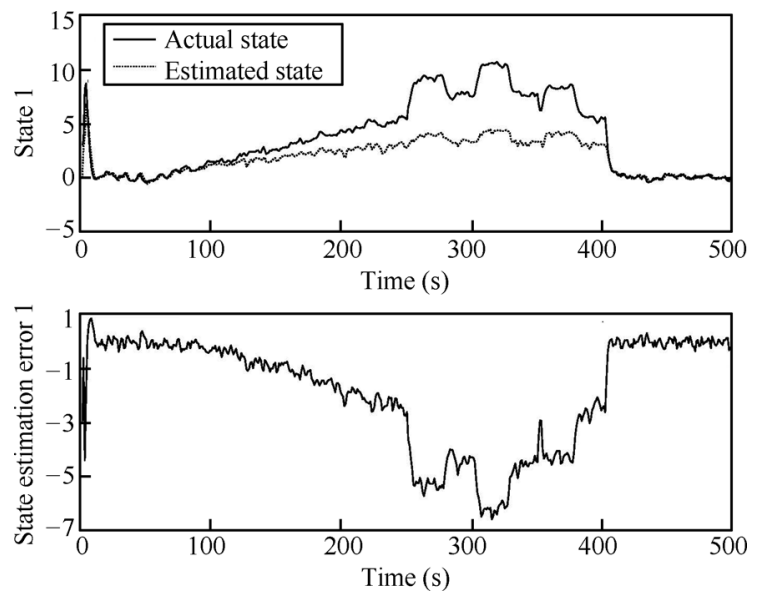

Fig. 4 Estimate of the first variable and its estimation error using the Kalman filter 
We also show the results of minimum square error unbiased estimation (MSUE) based filter in [4], which takes the knowledge of sensor missing measurement and unknown input dynamics into consideration, but overlooks the available knowledge on the unknown inputs. Similar technique to the proposed method 1 based filter has also been used to tackle the problem of multiple sensor missing measurements. The first state estimation (upper graphic) and estimation error (lower graphic) are shown in Fig. 5.
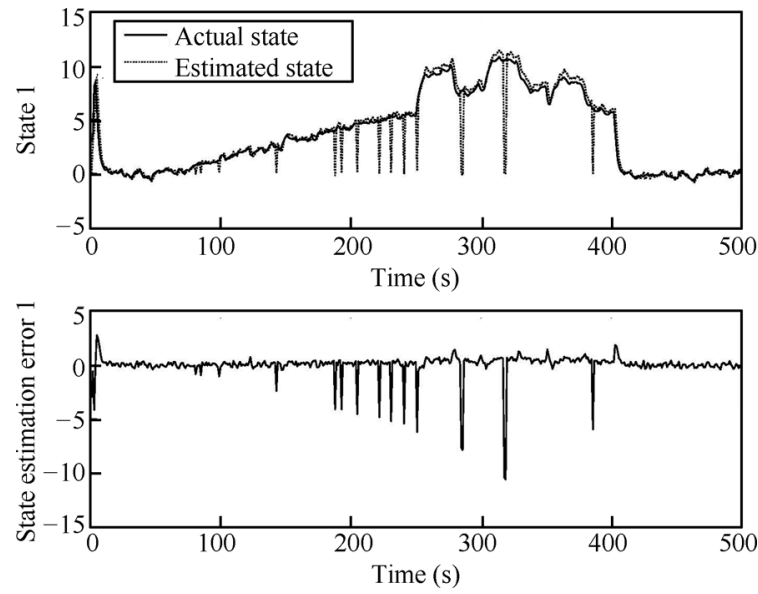

Fig. 5 Estimate of the first variable and its estimation error using the MSUE based filter

It can be seen from Fig. 5 that, by taking the unknown input dynamics into account, the MSUE based filter can obtain an unbiased estimation, which is obviously better than the biased results of the classical Kalman filter.

In the process of recursive filter derivation, the effect of $\left(\Xi_{k}-\bar{\Xi}_{k}\right) C_{k} x_{k}$ has been approximated using a Gaussian distribution with zero mean and covariance of $\operatorname{var}\left\{\left(\Xi_{k}-\right.\right.$ $\left.\left.\bar{\Xi}_{k}\right) C_{k} x_{k} \mid x_{k}\right\}$, although it generally does not follow a Gaussian distribution. The following Fig. 6 shows the approximation rationality by comparing the sample density function of $\left(\Xi_{k}-\bar{\Xi}_{k}\right) C_{k} x_{k}$ with the Gaussian probability density function used at some time step (only the first element of $x_{k}$ is shown).

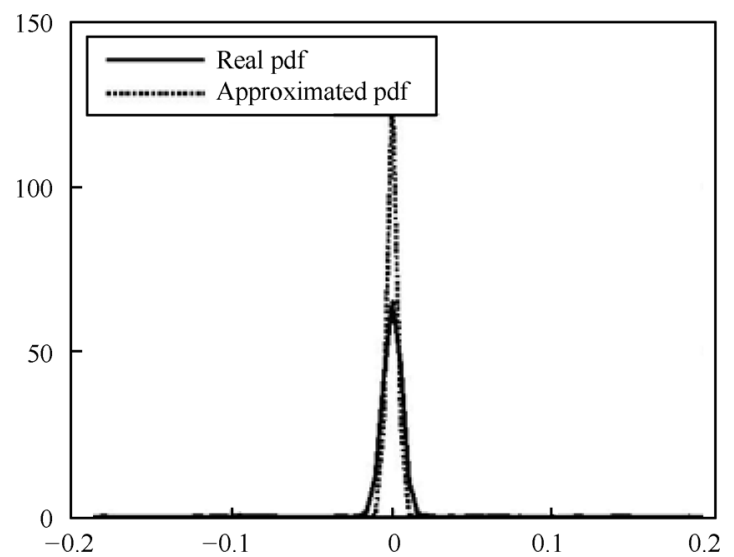

Fig. 6 Comparison between real and approximated pdfs

We further show the results of the proposed two meth- ods based filters. The simulation results are shown in Figs. 7 and 8, where the upper graphs of Figs. 7 and 8 display the simulated true values of the first state variable (real line) and the estimated state using the developed filters (dotted line), while the corresponding state estimation errors are shown in the lower graphs.
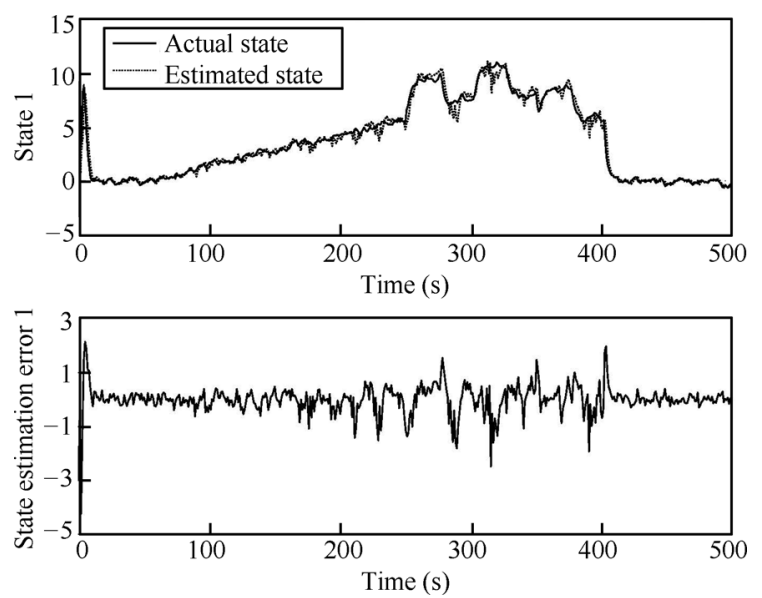

Fig. 7 Estimate of the first state variable and its estimation error using the proposed method 1 based filter
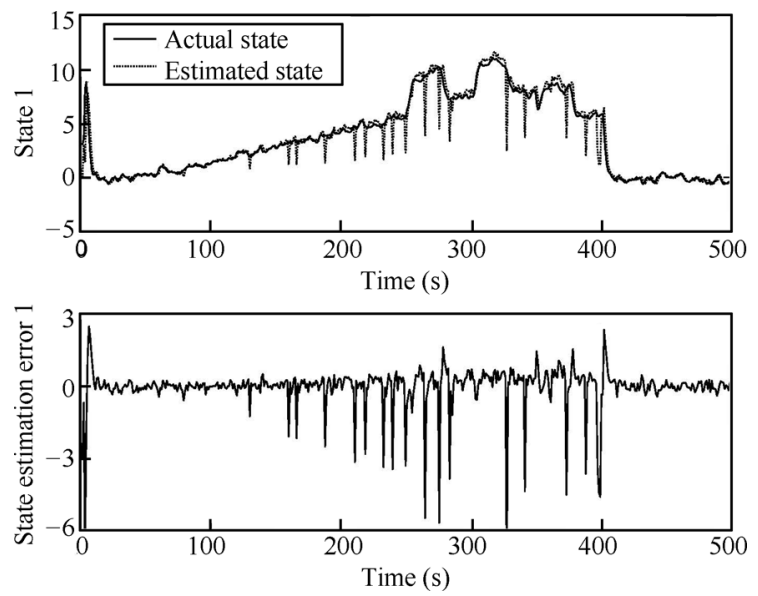

Fig. 8 Estimate of the first state variable and its estimation error using the proposed method 2 based filter

To evaluate the quality of the MSUE based filter and the proposed two methods based filters, we calculated the trace of the error covariance matrix $P_{k \mid k}$ at each time step and displayed it in Fig. 9.

It can be seen from Fig. 9 that the traces of the error covariance of the MSUE based filter and the developed two filters are bounded. The fluctuation of the trace of the error covariance is mainly due to the presence of partially observed inputs and missing measurements. In addition, the proposed two methods can obtain a better performance than the MSUE based filter. That is because the available knowledge on unknown inputs has been incorporated in our proposed method. It can also be obtained from Fig. 9 that the filter estimating $x_{k} x_{k}^{\mathrm{T}}$ based on $\mathrm{E}\left\{x_{k-1} x_{k-1}^{\mathrm{T}}\right\}$ (i.e., 
method 1) has better performance than the filter using the prior estimation of $x_{k}$ (i.e., method 2).

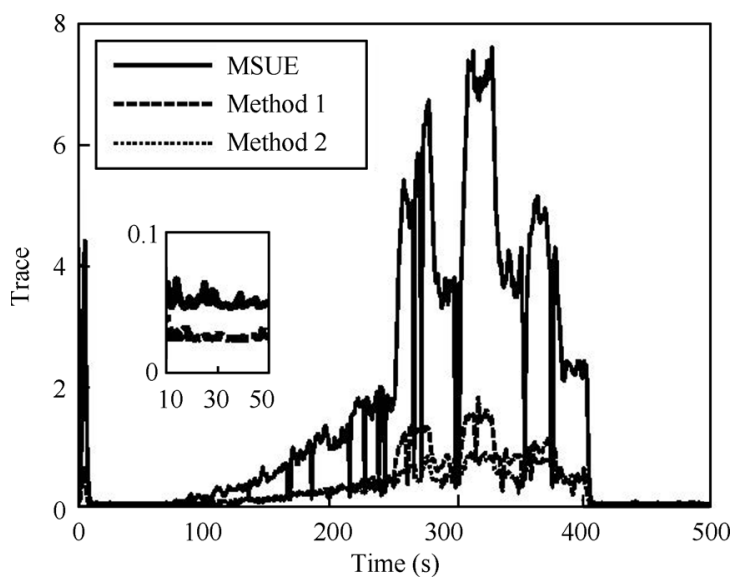

Fig. 9 Traces of the error covariance matrix $P_{k \mid k}$ for the three filters

\section{Conclusions}

The problem of state estimation is investigated in this paper for discrete-time linear systems, where knowledge is available on both unknown inputs and sensor missing measurements. First, the available knowledge on the unknown inputs and the state equations is used to form the prior distribution of the state vector at each step. Secondly, we approximate the measurement likelihood function by breaking down the effect of missing measurements and modelling the associated uncertainty as part of the measurement noise. Then, a recursive filter is obtained based on Bayesian inference which is suitable for online applications.

Simulation comparison results have been provided to show the performance improvement of the proposed method by incorporating available knowledge on unknown inputs. In future research, the main results of this paper could be extended to the filtering problem where the missing measurements also occur in the channel of partially observed inputs and possibly to the case of nonlinear systems based on particle filters.

\section{References}

[1] S. Kar, B. Sinopoli, J. M. F. Moura. Kalman filtering with intermittent observations: Weak convergence to a stationary distribution. IEEE Transactions on Automatic Control, vol. 57, no. 2, pp. 405-420, 2012.

[2] Y. Liang, T. W. Chen, Q. Pan. Optimal linear state estimator with multiple packet dropouts. IEEE Transactions on Automatic Control, vol. 55, no. 6, pp. 1428-1443, 2010.

[3] B. Li. A non-Gaussian Kalman filter with application to the estimation of vehicular speed. Technometrics, vol. 51, no. 2, pp. 162-172, 2009.

[4] P. K. Kitanidis. Unbiased minimum-variance linear state estimation. Automatica, vol. 23, no. 6, pp. 775-778, 1987.
[5] F. O. Hounkpevi, E. F. Yaz. Robust minimum variance linear state estimators for multiple sensors with different failure rates. Automatica, vol. 43, no. 7, pp. 1274-1280, 2007.

[6] J. Hu, Z. D. Wang, H. J. Gao, L. K. Stergiouls. Extended Kalman filtering with stochastic nonlinearities and multiple missing measurements. Automatica, vol. 48, no. 9, 20072015, 2012.

[7] Z. D. Wang, D. W. C. Ho, X. H. Liu. Variance-constrained filtering for uncertain stochastic systems with missing measurements. IEEE Transactions on Automatic Control, vol. 48, no. 7, pp. 1254-1258, 2003.

[8] X. M. Qu, J. Zhou. The optimal robust finite-horizon Kalman filtering for multiple sensors with different stochastic failure rates. Applied Mathematics Letters, vol. 26, no. 1, pp. 80-86, 2013.

[9] H. Y. Liang, T. Zhou. Robust state estimation for uncertain discrete-time stochastic systems with missing measurements. Automatica, vol. 47, no. 7, pp. 1520-1524, 2011.

[10] S. L. Sun, L. H. Xie, W. D. Xiao, Y. C. Soh. Optimal linear estimation for systems with multiple packet dropouts. Automatica, vol. 44, no. 5, pp. 1333-1342, 2008.

[11] S. L. Sun, L. H. Xie, W. D. Xiao. Optimal full-order and reduced-order estimators for discrete-time systems with multiple packet dropouts. IEEE Transactions on Signal Processing, vol. 56, no. 8, pp. 4031-4038, 2009.

[12] B. Li. State estimation with partially observed inputs: A unified Kalman filtering approach. Automatica, vol. 49, no. 3, pp. 816-820, 2013.

[13] W. H. Chen, D. J. Ballance, P. J. Gawthrop, J. O'Reilly. A nonlinear disturbance observer for robotic manipulators. IEEE Transactions on Industrial Electronics, vol. 47, no. 4 , pp. 932-938, 2000.

[14] J. Yang, S. H. Li, J. Y. Su, X. H. Yu. Continuous nonsingular terminal sliding mode control for systems with mismatched disturbances. Automatica, vol. 49, no. 7, pp. 22872291, 2013.

[15] J. Y. Su, J. Yang, S. H. Li. Continuous finite-time antidisturbance control for a class of uncertain nonlinear systems. Transactions of the Institute of Measurement and Control, vol.36, no. 3, pp. 300-311, 2014.

[16] J. Yang, J. Su, S. Li, X. Yu. High-order mismatched disturbance compensation for motion control system via a continuous dynamic sliding-mode approach. IEEE Transactions on Industrial Informatics, vol. 10, no. 1, pp.604-614, 2014.

[17] Y. Cheng, H. Ye, Y. Q. Wang, D. H. Zhou. Unbiased minimum-variance state estimation for linear systems with unknown input. Automatica, vol. 45, no. 2, pp. 485-491, 2009 .

[18] S. Gillijns, B. De Moor. Unbiased minimum-variance input and state estimation for linear discrete-time systems. $A u-$ tomatica, vol. 43, no. 1, pp.111-116, 2007.

[19] D. Simon. Optimal State Estimation: Kalman, H Infinity, and Nonlinear Approaches, New York, USA: Wiley, 2006. 
[20] J. Y. Su, B. B. Li, W. Chen. Bayesian recursive filtering with partially observed inputs and missing measurements. In Proceedings of the 19th International Conference on $\mathrm{Au}-$ tomation and Computing, IEEE, Uxbridge, UK, pp.1-6, 2013.

[21] R. A. Horn, C. R. Johnson. Topics in Matrix Analysis, Cambridge, UK: Cambridge University Press.

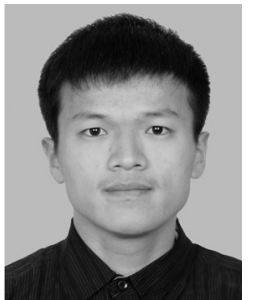

Jinya Su received the B. Sc. degree in the School of Mathematics and Statistics from Shandong University, China in 2011. He is currently a Ph. D. candidate in Department of Aeronautical and Automotive Engineering, Loughborough University, Loughborough, UK.

His research interests include disturbance observer design and its applications in fault diagnosis and disturbance rejection control.

E-mail: J.Su2@lboro.ac.uk (Corresponding author)

ORCID iD: 0000-0002-3121-7208

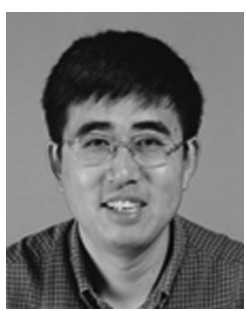

Baibing Li received the B.Sc. degree from Yunnan University, China, the M. Sc. degree from Shanghai Jiao Tong University, China, and the M.Sc. degree from Vrije Universiteit Brussel, Belgium. In 1991, he received the $\mathrm{Ph}$. D. degree from the Management School, Shanghai Jiao Tong University. He was a postdoctoral research fellow with Katholieke Universiteit Leuven, Belgium, and a research associate with Newcastle University,
Newcastle upon Tyne, UK. In 2001, he was appointed as a lecturer at Newcastle University. In 2004, he moved to the School of Business and Economics, Loughborough University, Loughborough, UK, as a Lecturer, where he was subsequently appointed as a reader in 2007 and a professor in 2011. He is a member of IEEE and the Royal Statistical Society.

His current research interests include Bayesian statistical modeling and forecasting for Gaussian and non-Gaussian dynamic problems in various management areas, transportation demand analysis, travel behavior modeling, and intelligent transportation systems.

E-mail: b.li2@lboro.ac.uk

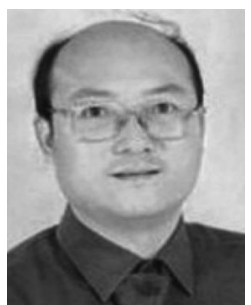

Wen-Hua Chen received the M. Sc. and $\mathrm{Ph}$. D. degrees from Northeast University, China in 1989 and 1991, respectively. From 1991 to 1996, he was a lecturer and then an associate professor with the Department of Automatic Control, Nanjing University of Aeronautics and Astronautics, China. From 1997 to 2000, he held a research position and then a lecturer in control engineering with the Centre for Systems and Control, University of Glasgow, UK. In 2000, he moved to the Department of Aeronautical and Automotive Engineering, Loughborough University, UK, as a lecturer, where he was appointed as a professor in 2012. He is a senior member of IEEE and a Fellow of IET.

His research interests include the development of advanced control strategies (nonlinear model predictive control, disturbance observer based control, etc.) and their applications in aerospace engineering and the development of unmanned autonomous intelligent systems.

E-mail: W.Chen@lboro.ac.uk 